\title{
Multimodal Interaction for Data Visualization
}

\author{
Bongshin Lee \\ Microsoft Research \\ Redmond, WA, USA \\ bongshin@microsoft.com
}

\author{
Arjun Srinivasan, John Stasko \\ Georgia Institute of Technology \\ Atlanta, GA, USA \\ \{arjun010,stasko\}@gatech.edu
}

\author{
Melanie Tory, Vidya Setlur \\ Tableau Software \\ Palo Alto, CA, USA \\ \{mtory,vsetlur\}@tableau.com
}

\begin{abstract}
Multimodal interaction offers many potential benefits for data visualization. It can help people stay in the flow of their visual analysis and presentation, with the strengths of one interaction modality offsetting the weaknesses of others. Furthermore, multimodal interaction offers strong promise for leveraging data visualization on diverse display hardware including mobile, AR/VR, and large displays. However, prior research on visualization and interaction techniques has mostly explored a single input modality such as mouse, touch, pen, or more recently, natural language. The unique challenges and opportunities of synergistic multimodal interaction for data visualization have yet to be investigated. This workshop will bring together researchers with expertise in visualization, interaction design, and natural user interfaces. We aim to build a community of researchers focusing on multimodal interaction for data visualization, explore opportunities and challenges in our research, and establish an agenda for multimodal interaction research specifically for data visualization.
\end{abstract}

\section{CCS CONCEPTS}

\section{- Human-centered computing $\rightarrow$ Visualization; Interaction paradigms;}

\section{KEYWORDS}

Data Visuallization, Multimodal Interaction, Interaction Design

\section{ACM Reference Format:}

Bongshin Lee, Arjun Srinivasan, John Stasko, and Melanie Tory, Vidya Setlur. 2018. Multimodal Interaction for Data Visualization. In AVI '18: 2018 International Conference on Advanced Visual Interfaces, AVI '18, May 29-fune 1, 2018, Castiglione della Pescaia, Italy. ACM, New York, NY, USA, Article 4, 3 pages. https://doi.org/10.1145/3206505.3206602

\section{INTRODUCTION}

Data visualization systems have predominantly been developed for windows, icons, menus, pointer (WIMP) based interfaces. More recently, there has been increased interest within the visualization community to explore visualizations on devices and settings where conventional input modalities such as keyboard and mouse are not available [12]. Such interfaces, commonly referred to as post-WIMP interfaces, have explored a variety of input modalities including touch $[15,19]$, mid-air gestures [1, 9], and natural

Permission to make digital or hard copies of part or all of this work for personal or classroom use is granted without fee provided that copies are not made or distributed for profit or commercial advantage and that copies bear this notice and the full citation on the first page. Copyrights for third-party components of this work must be honored.

For all other uses, contact the owner/author(s).

AVI '18, May 29-fune 1, 2018, Castiglione della Pescaia, Italy

(c) 2018 Copyright held by the owner/author(s).

ACM ISBN 978-1-4503-5616-9/18/05.

https://doi.org/10.1145/3206505.3206602 language $[4,16,17]$, among others. Multimodal interaction offers many potential benefits for data visualization to help people stay in the flow of their visual analysis and presentation $[6,18]$. Often, the strengths of one interaction modality can offset weaknesses of another. However, existing visualization tools and interaction techniques have mostly explored the aforementioned forms of input as a single input modality. Recent interest in deploying data visualizations on diverse display hardware including mobile, AR/VR, and large displays create an urgent need to develop natural and fluid interaction techniques that can work in these contexts. Multimodal interaction offers strong promise for such situations, but its unique challenges for data visualization have yet to be deeply investigated.

The goal of this workshop is to bring together researchers with expertise in visualization, interaction design, and natural user interfaces. We aim to build a community of multimodal visualization researchers, explore synergies and challenges in our research, and establish an agenda for research on multimodal interactions for visualization.

\section{WORKSHOP INFORMATION}

\subsection{Previous Related Workshops}

Recognizing the advancement of interaction technologies and the potential benefits they bring to data visualization, research communities have started to pay increasing attention to leveraging them for data visualization. Two DEXIS (Visual Data Exploration on Interactive Surfaces) workshops - DEXIS 2011 and 2015, explored the research space on how to leverage interactive tabletops and surfaces for data visualization. Two immersive analytics workshops held at ISS 2016 and VIS 2017 called for exploration on the applicability and development of emerging user-interface technologies for creating more engaging experiences for data analysis and presentation. In addition, the CHI 2018 Workshop on Data Visualization on Mobile Devices specifically focuses on how to leverage ubiquitous mobile devices as data visualization platforms. However, all the prior workshops focused mainly on touch and gestures. The prevalence of natural language being a promising medium for people to intuitively converse with their data, provides interesting and exciting opportunities for future research. Our workshop focuses on natural language interaction as a core topic, with the potential synergy that would come from effectively combining multiple interaction modalities. Thus, it will complement the previous and ongoing workshops held to develop new research directions that advance data visualization and interaction.

\subsection{Workshop Activities}

The full-day workshop was structured to provide maximum time for group discussion and brainstorming. The schedule of events is as follows: 


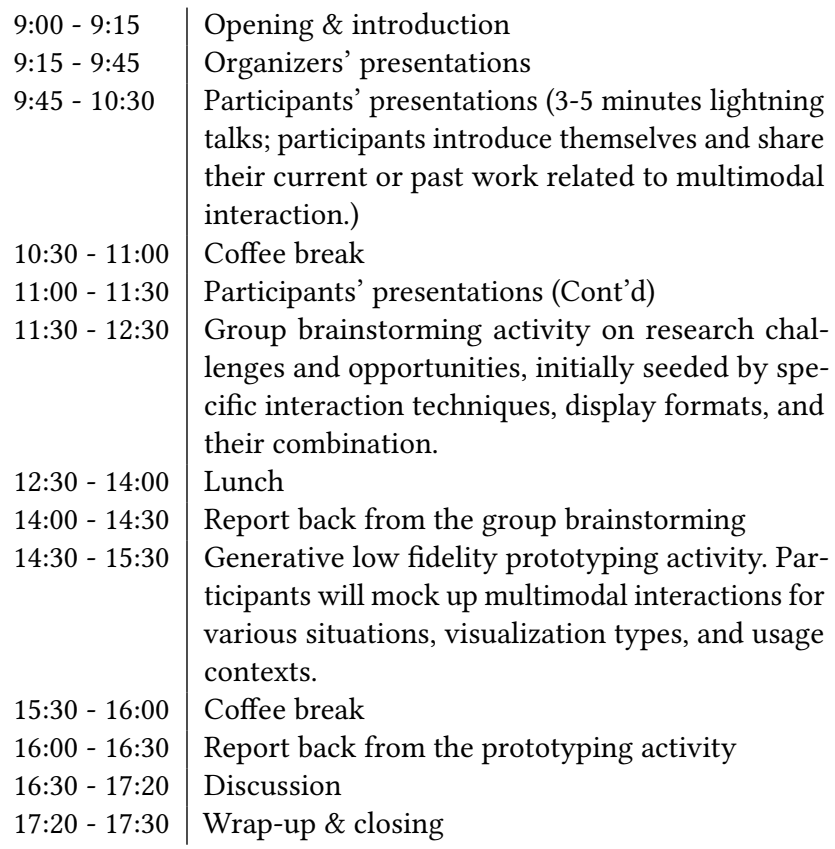

\section{SUBMISSION ABSTRACTS}

A total of nine submissions were accepted as part of the workshop proceedings. The submissions spanned a variety of topics discussing a range of input modalities including speech, touch, gestures, and even smell. The accepted submissions along with the respective authors and abstracts are provided below.

\section{Combining Interactive Large Displays and Smartphones to} Enable Data Analysis from Varying Distances [11]

Ricardo Langner, Ulrike Kister, Marc Satkowski and Raimund Dachselt. Interactive Media Lab, Technische Universität Dresden.

Large high-resolution displays (LHRD) can benefit information visualization due to their size and resolution. As users often move back and forth in front of the display, we need to investigate what tasks should be supported from varying distances. In this work, we combine touch interaction on a LHRD with casual, 'eyes-free' device interaction using smartphones from a distance. We describe early insights and discuss important questions based on experiences with our prototype implementation.

\section{A Novel Interaction Paradigm For Exploring Spatio-Temporal Data [2] \\ Sabine Cassat, Marcos Serrano, Emmanuel Dubois and Pourang Irani \\ University of Toulouse, IRIT and University of Manitoba \\ Complex spatio-temporal data is difficult to visualize and even fur- ther to interact with, especially by several users at the same time. However, visualization and exploration of such data are essential for experts to understand complex data environments, such as for mitigating the adverse effects of disease spread. This paper presents an alternative approach to that of current spatiotemporal data vi- sualizations to access, interpret, and manipulate spatio-temporal datasets as a single user or as a team. Our approach uses tangible and}

visual tools such as mini-robots, tabletop displays and augmented reality tools, to facilitate the data exploration and interpretation. We also introduce a simple use case that illustrates one of the possible utilization of the system. While tangibles have been introduced to represent information, we are investigating manners in which we can depict even more complex datasets. Our system will provide a novel approach to manipulate $3 \mathrm{D}$ and $4 \mathrm{D}$ datasets that classic tools such as a 2D mouse or a tactile screen would not allow.

Gaze-Enabled Data Recommendations In Visualization: First Considerations [8]

Radu Jianu

City, University of London

Cheaper and better eye-trackers now make it possible to use this technology to determine users' data interests in real-time, as they visually explore large and heterogeneous data spaces. Data visualizations could use such knowledge to continuously adapt and show data that is well aligned with users' interests and goals. Recommendation systems based on logging and interpreting manual interactions have been studied already. However, integrating eye-tracking into the recommendation process is important in the visualization domain given the medium's inherently visual nature and it poses unique challenges and questions. We introduce and discuss these aspects as they arose from an informal, exploratory investigation.

\section{Olfactory Analytics: Exploring the Design Space of Smell for Data Visualization [13] \\ Biswaksen Patnaik, Andrea Batch and Niklas Elmqvist University of Maryland}

The use of olfactory feedback for analytical tasks is a virtually unexplored area in spite of the advantages it offers for information recall, feature identification, and location detection. In this paper, we introduce the concept of information olfactation as the fragrant sibling of information visualization, and propose some olfactory channels for analysis. To exemplify this idea, we present our prototype system combining smell with information visualization, with use cases in 2D graph visualization as well as in virtual reality.

Toward Multimodal Interaction of Scatterplot Spaces Exploration [3]

Mohammad Chegini, Lin Shao, Keith Andrews and Tobias Schreck Graz University of Technology

The latest generation of large vertically-mounted multitouch displays bring new opportunities for solving visual analytics tasks. Due to their size, it is possible to visualise and collaboratively interact with high-dimensional datasets and multiple views (e.g., scatterplots, scatterplot matrices and parallel coordinates). However, using only multi-touch for input can be overly restrictive. Other modalities need to be considered to utilise the power of these screens fully. By adding natural language interaction, the user can directly interact with the visual analytics application from a distance. Incorporating eye-tracking can help narrow down what the user is looking at or is interested in. In this paper, some of the challenges of using multi-touch as input for the analysis of scatterplot spaces on large vertically-mounted multitouch displays are discussed and addressed by proposing the incorporation of other interaction modalities. 


\section{Employing Tangible Visualisations in Augmented Reality with} Mobile Devices [7]

Sebastian Hubenschmid, Simon Butscher, Johannes Zagermann and Harald Reiterer

HCI Group, University of Konstanz

Recent research has demonstrated the benefits of mixed realities for information visualisation. Often the focus lies on the visualisation itself, leaving interaction opportunities through different modalities largely unexplored. Yet, mixed reality in particular can benefit from a combination of different modalities. This work examines an existing mixed reality visualisation which is combined with a large tabletop for touch interaction. Although this allows for familiar operation, the approach comes with some limitations which we address by employing mobile devices, thus adding tangibility and proxemics as input modalities.

Towards Multimodal Data Analytics: Integrating Natural Language into Visual Analytics [5]

Rafael Henkin and Cagatay Turkay giCentre, City, University of London

The continuous interaction between users and the system in visual analytics can be considered a dialogue. We propose the use of multiple two-way channels facilitated by a multimodal interface as a central aspect of interactive visualization design, in particular, the use of natural language with interactive visualization. We discuss key related concepts, potential benefits, challenges and opportunities that emerge as a research agenda for multimodal data analysis.

Bridging the Gap Between Sonification and Visualization [14] Alexander Rind, Michael Iber and Wolfgang Aigner

St. Pölten University of Applied Sciences, Austria

Extensive research has been carried out both on auditory and visual representation of data. Still, there is huge potential for complementary audio-visual analytics environments. This position paper works towards a research agenda for interdisciplinary work.

Personal Data Exploration with Speech on Mobile Devices [10] Eun Kyoung Choe, Bongshin Lee and Seung-Won Hwang University of Maryland, Microsoft Research, and Yonsei University

In this article, we envision a novel way to help people explore their personal data on mobile devices by leveraging speech interaction. Supporting visual data exploration on mobile devices pertains to two constraints. First, mobile devices' small screen cannot afford a panel of widgets. Second, exploring self-tracking data often involves entering specific date and time, or their ranges, which is tedious to do on mobile devices. These challenges can be addressed through speech because it does not require much space and is flexible enough to cover different ways to specify date and time. Moreover, common personal insight types identified from prior work allow us to narrow the scope of the speech interaction needed for personal data exploration, making it more tractable with the state-of-the-art speech recognition technology.

\section{REFERENCES}

[1] S. K. Badam, F. Amini, N. Elmqvist, and P. Irani. 2016. Supporting visual exploration for multiple users in large display environments. In 2016 IEEE Conference on Visual Analytics Science and Technology (VAST). 1-10. https: //doi.org/10.1109/VAST.2016.7883506

[2] Sabine Cassat, Marcos Serrano, Emmanuel Dubois, and Pourang Irani. 2018. A Novel Interaction Paradigm For Exploring Spatio-Temporal Data. AVI 2018 Workshop on Multimodal Interaction for Data Visualization (May 2018). https: //multimodalvis.github.io/papers/AVI_2018_paper_146.pdf

[3] Mohammad Chegini, Lin Shao, Keith Andrews, and Tobias Schreck. 2018. Toward Multimodal Interaction of Scatterplot Spaces Exploration. AVI 2018 Workshop on Multimodal Interaction for Data Visualization (May 2018). https://multimodalvis. github.io/papers/AVI_2018 paper_149.pdf

[4] Tong Gao, Mira Dontcheva, Eytan Adar, Zhicheng Liu, and Karrie G Karahalios. 2015. Datatone: Managing ambiguity in natural language interfaces for data visualization. In Proceedings of the UIST. 489-500.

[5] Rafael Henkin and Cagatay Turkay. 2018. Towards Multimodal Data Analytics: Integrating Natural Language into Visual Analytics. AVI 2018 Workshop on Multimodal Interaction for Data Visualization (May 2018). https://multimodalvis. github.io/papers/AVI_2018_paper_152.pdf

[6] Enamul Hoque, Vidya Setlur, Melanie Tory, and Isaac Dykeman. 2018. Applying Pragmatics Principles for Interaction with Visual Analytics. IEEE transactions on visualization and computer graphics 24,1 (2018), 309-318.

[7] Sebastian Hubenschmid, Simon Butscher, Johannes Zagermann, and Harald Reiterer. 2018. Employing Tangible Visualisations in Augmented Reality with Mobile Devices. AVI 2018 Workshop on Multimodal Interaction for Data Visualization (May 2018). https://multimodalvis.github.io/papers/AVI_2018_paper_150.pdf

[8] Radu Jianu. 2018. Gaze-Enabled Data Recommendations In Visualization: First Considerations. AVI 2018 Workshop on Multimodal Interaction for Data Visualization (May 2018). https://multimodalvis.github.io/papers/AVI_2018_paper_147. pdf

[9] Ulrike Kister, Patrick Reipschläger, Fabrice Matulic, and Raimund Dachselt. 2015. BodyLenses: Embodied Magic Lenses and Personal Territories for Wall Displays. In Proceedings of the 2015 International Conference on Interactive Tabletops \& Surfaces (ITS '15). ACM, 117-126. https://doi.org/10.1145/2817721.2817726

[10] Eun Kyoung Choe, Bongshin Lee, and Seung-Won Hwang. 2018. Personal Data Exploration with Speech on Mobile Devices. AVI 2018 Workshop on Multimodal Interaction for Data Visualization (May 2018). https://multimodalvis.github.io/ papers/AVI_2018_paper_159.pdf

[11] Ricardo Langner, Ulrike Kister, Marc Satkowski, and Raimund Dachselt. 2018. Combining Interactive Large Displays and Smartphones to Enable Data Analysis from Varying Distances. AVI 2018 Workshop on Multimodal Interaction for Data Visualization (May 2018). https://multimodalvis.github.io/papers/AVI_2018_ paper_145.pdf

[12] Bongshin Lee, Petra Isenberg, Nathalie Henry Riche, and Sheelagh Carpendale. 2012. Beyond mouse and keyboard: Expanding design considerations for information visualization interactions. IEEE Transactions on Visualization and Computer Graphics 18, 12 (2012), 2689-2698.

[13] Biswaksen Patnaik, Andrea Batch, and Niklas Elmqvist. 2018. Olfactory Analytics: Exploring the Design Space of Smell for Data Visualization. AVI 2018 Workshop on Multimodal Interaction for Data Visualization (May 2018). https://multimodalvis. github.io/papers/AVI_2018_paper_148.pdf

[14] Alexander Rind, Michael Iber, and Wolfgang Aigner. 2018. Bridging the Gap Between Sonification and Visualization. AVI 2018 Workshop on Multimodal Interaction for Data Visualization (May 2018). https://multimodalvis.github.io/ papers/AVI_2018_paper_155.pdf

[15] Ramik Sadana and John Stasko. 2016. Designing multiple coordinated visualizations for tablets. In Computer Graphics Forum, Vol. 35. Wiley Online Library, 261-270.

[16] Vidya Setlur, Sarah E Battersby, Melanie Tory, Rich Gossweiler, and Angel X Chang. 2016. Eviza: A natural language interface for visual analysis. In Proceedings of the UIST. 365-377.

[17] Arjun Srinivasan and John Stasko. 2017. Natural Language Interfaces for Data Analysis with Visualization: Considering What Has and Could Be Asked. EuroVis - Short Papers (Jun 2017), 55-59. https://doi.org/10.2312/eurovisshort.20171133

[18] Arjun Srinivasan and John Stasko. 2018. Orko: Facilitating Multimodal Interaction for Visual Exploration and Analysis of Networks. IEEE transactions on visualization and computer graphics 24,1 (2018), 511-521.

[19] Jagoda Walny, Bongshin Lee, Paul Johns, Nathalie Henry Riche, and Sheelagh Carpendale. 2012. Understanding pen and touch interaction for data exploration on interactive whiteboards. IEEE Transactions on Visualization and Computer Graphics 18, 12 (2012), 2779-2788. 